\title{
DIE KONDITIONALE ANALYSE DES PRAKTISCHEN KÖNNENS
}

Gottfried SEEBASS

Universität Konstanz

„I will not do't.“

„But can you, if you would?"

"Look, what I will not, that I cannot do."

(Shakespeare: Measure for Measure II,2)

\section{Der Gattungsbegriff des Könnens}

Einer der Gründe, die Aristoteles als einen der ersten sprachanalytischen Philosophen erscheinen lassen, ist sein Bemühen, Mehrdeutigkeiten und verborgene semantische Zusammenhänge herauszuarbeiten. Betroffen davon sind auch die Modalausdrücke ,,möglich“" und ,,notwendig“, , ,können“ und ,,müssen“. In der ,,Metaphy$\operatorname{sik}^{\prime \prime}(\mathrm{V}, 5)$ unterscheidet Aristoteles u.a. zwischen der logischen und der kausalen Bedeutung des Wortes ,„notwendig“" (,anangkaion“), sowie der Notwendigkeit des Zwangs. Gleichzeitig aber führt er all diese Verwendungsweisen auf eine zurück. „Notwendig“" sei das, wozu es keine Alternativen gibt, oder, wie Aristoteles etwas ausführlicher formuliert, ,,was sich nicht anders verhalten kann" (,, to me endechomenon allos echein").

Ich denke, damit hat er die Sache getroffen. Und die Richtigkeit der Aristotelischen Begriffsbestimmung wird dadurch weiter gestiitzt, daß sie uns in die Lage versetzt, neben der alethischen und der praktisch rationalen auch die deontische Rede vom ,Notwendigsein“ oder ,,Müssen“ verständlich zu machen. Sätze wie ,Kraftfahrer müssen die rechte Straßenseite benutzen“, ,Gipfelstürmer müssen früh aufstehen“, ,,Junggesellen müssen unverheiratet sein“" oder , Schnittblumen müssen welken“ kommen, so verschieden ihre Rede vom „Müssen“ ist, doch in zwei Punkten überein: jeder dieser Sätze nimmt (1) auf einen Spielraum denkbarer Alternativen Bezug, 
von denen er (2) behauptet, daß sie verschlossen sind außer der einen, die wegen dieser Einzigkeit ,,notwendig" ist. Spezielle Notwendigkeitsbegriffe unterscheiden sich durch die Art ihrer Alternativen und durch die Gründe, die dafür verantwortlich sind, daß sie als offen oder verschlossen gelten. Beim deontischen Müssen, für das mein Kraftfahrerbeispiel steht, bilden Handlungen die Alternativen und Normen den Grund. Beim kausalen Müssen wiederum, das mein Blumenbeispiel exemplifiziert, werden nicht handlungshafte Ereignisse als Alternativen ausgeschlossen, relativ auf die Kausalgesetze und die bestehenden Kausalbedingungen. Und so weiter.

Der Vielfalt spezifizierter Notwendigkeitsbegriffe korrespondiert eine ebenso große Vielfalt der Begriffe von Möglichkeit. Beide sind interdefiniert. Daß etwas ,,möglich“ ist oder sein ,,kann“, bedeutet nach der kolloquialen, engeren Auffassung, daß es weder notwendig ist noch notwendig nicht ist, d.h. daß die Alternativen des Seins wie die des Nichtseins hier nicht verschlossen sind.' Vom

1. Kolloquial wird niemand etwas, das er für notwendig hält, zugleich als möglich ansprechen. Modallogisch jedoch schließt Mp bekanntlich nur - N-p aus, umfaßt also Np genauso wie Fp (= faktisch p) oder F-p. Man könnte daraus den Schluß ziehen, daß die Aristotelische Bedingung, $\mathrm{da} B$ mehrere Alternativen offen sein müssen, hier nicht greift, da die Alternative des Nichtbestehens von $p$ im Falle von $\mathrm{Np}$ und sogar $\mathrm{Fp}$, falls wir fixierte Wahrheitswerte zugrundelegen, verschlossen ist. Doch anstatt das Konzept zu widerlegen, bestätigt der Einwand nur, wie wichtig es ist, verschiedene Verwendungsweisen der Ausdrücke ,,möglich“ und ,notwendig“ auseinanderzuhalten. Entweder nämlich ist das inklusiv gedeutete ,Mp“ nur ein logischer Kunstausdruck, der mit der gewöhnlichen Rede vom ,Möglichsein“ wenig zu tun hat, weniger noch als (z.B.) das materiale Konditional der Aussagenlogik mit normalen Wenn-dann-Sätzen. Oder aber es hat den gewöhnlichen Sinn und bezieht sich undifferenziert auf alle Alternativen, die nach dem Ausscheiden von $-\mathrm{N}$-p, das hier allein als verschlossen gilt, offen bleiben, sc. F-p, Fp und Np.

Kritisch für das Aristotelische Konzept ist allenfalls die Notwendigkeit des Prinzips der Widerspruchsfreiheit. Denn es ist mehr als zweifelhaft, daß sich ,p \& - $\mathrm{p}$ “ überhaupt als eine ,--(p \& -p)" korrespondierende denkbare Alternative begreifen läßt, die offen oder verschlossen sein kann. Muß man deshalb, um die Generalität des Konzepts zu retten, den Schritt zur ,,dialethischen Logik“ tun? Nein, denn es gibt eine plausible konzeptionelle Alternative. Der „Satz vom Widerspruch" wird danach nicht mehr als Satz aufgefaßt, der notwendig wahr ist, 
„Möglichsein“ oder ,,Können“ läßt sich kolloquial nur reden, wenn nicht nur eine der denkbaren Alternativen als offen gilt, sondern mindestens zwei, was im Grenzfall, in dem ein einziges Paar kontradiktorischer Alternativen A/-A zugrundeliegt, mit der völligen Offenheit des Spielraums zusammenfällt. ${ }^{2}$ Die Rede vom ,,Können“ aber, so scheint es, ist konstitutiv für den Freiheitsbegriff. Folglich wird er von der modalen Differenzierung mitberührt. Um zu verstehen, was Freiheit ist, müssen wir Klarheit darüber gewinnen, in welchem Sinne wir hier vom „Können“ reden. Dem gilt mein Interesse in diesem Aufsatz.

\section{Freiheit als Möglichkeitsbegriff}

Zuvor allerdings stellt sich die Frage, ob der Begriff der Freiheit tatsächlich nur als ein Möglichkeitsbegriff zu verstehen ist. Daß es modale Verwendungsweisen der Wörter ,,frei“" oder ,,Freiheit" gibt, wird niemand in Abrede stellen. Das prominenteste Beispiel liefert der klassische Begriff der ,Handlungsfreiheit“, nach dem frei zu sein soviel bedeutet wie handeln zu können, wie man will. Die Frage ist nur, ob unsere Rede von ,Freiheit“ ausnahmslos oder im Kern eine modal qualifizierte ist.

Teilweise wird das bestritten. In der Diskussion um die politische Freiheit etwa wird häufig geltend gemacht, es genüge nicht, Freiheitsrechte zu etablieren, die jedem Bürger die Möglichkeit geben, bestimmte Interessen frei von gesetzlichen Restriktionen und staat-

sondern als Metaregel, die jeder Verwendung wahrheitsfähiger Sätze vorausliegt. Der umfassende Spielraum von Alternativen wird eben durch die Gesamtheit dessen gebildet, was denkbar ist, und das heißt offenbar widerspruchsfrei denkbar. Vgl. dazu auch G. Seebaß: Wollen, Frankfurt 1993, 96f. Anm. 159-163, 140 Anm. 213.

2. Der Grenzfall ist selten bzw. ein Resultat theoretischer Abstraktion und Vereinfachung. Normalerweise werden die Alternativen durch Kombinationen von Sachverhalten gebildet, wenn auch (normalerweise) keine vollständigen „,möglichen Welten“ oder ,Weltzustände“. Hier also gibt es partiell offene und verschlossene Spielräume. Wenn etwa $q$ eine notwendige Bedingung für $p$ ist, $p$ aber nicht umgekehrt auch für $q$, und wenn beide, $q$ wie $p$, prinzipiell möglich sind, sind die Alternativen $\mathrm{p} \& \mathrm{q} / \mathrm{p}$ \& $\mathrm{q} / \mathrm{-p}$ \& $-\mathrm{q}$ offen, während $\mathrm{p} \&$-q verschlossen ist. Und so weiter. 
lichen oder privaten Interventionen zu verfolgen; hinzukommen müsse der aktuelle Besitz geeigneter Fähigkeiten und Mittel, um den politischen Handlungsspielraum zu nutzen. Menschen, die aufgrund ihrer sozialen Stellung, mangelnden Ausbildung oder körperlichen und geistigen Behinderung nicht in der Lage sind, ihre wahren Interessen zu erkennen oder zu realisieren, sind in einem signifikanten Sinn unfrei, obwohl auch sie prinzipiell über dieselben Optionen verfügen wie alle. Charles Taylor vor allem hat diesen Begriff der ,positiven Freiheit" gegen Isaiah Berlin und andere Vertreter (liberalistischer) ,negativer" Freiheitskonzepte verteidigt. $^{3}$

Allerdings wird der modale Freiheitsbegriff hiẹr nur ergänzt, nicht verabschiedet. Doch könnte man nicht noch einen Schritt weiter gehen und den ,Verwirklichungsbegriff“ (Taylor) der Freiheit ganz an die Stelle des Möglichkeitsbegriffes setzen? Manches, was Aristoteles über Freiheit sagt, könnte das nahelegen. Denn ihm zufolge ist eine Handlung dann ,,freiwillig"(,,hekon“, ,,hekusios"), wenn bzw. soweit der Handelnde wei $\beta$, was er tut, und ohne Zwang handelt, wobei das negative. Kriterium der Zwanglosigkeit durch zwei positive Kriterien erläutert wird: (a) daß der Ursprung der Handlung im Handelnden liegt (,,arche en auto") und (b) daß er derjenige ist, bei dem es steht (,,ep' auto"), ob sie eintritt oder nicht. ${ }^{4}$ Der geforderte Zustand des Wissens jedoch und der Handlungsbeginn im Handelnden sind Tatsachen, nicht bloße Möglichkeiten. Sollte man sich deshalb auf diese Merkmale des Aristotelischen Freiheitsbegriffs beschränken können, bräuchte man sich um dessen modalen Sinn nicht mehr zu kümmern. Wer z.B. (mit Wittgenstein oder Ryle) der Meinung ist, daß die Rede von ,Freiheit“ insgesamt auf die von der ,,Freiwilligkeit" von Handlungen zurückführt und daß diese nur in bestimmten Umständen besteht, unter denen die Handlungen ausgeführt werden, kann die Aristotelischen Kriterien

3. I. Berlin: ,Two concepts of liberty“, [orig. 1958] repr. in: (ders.:) Four essays on liberty, Oxford 1969, 118-172; Ch. Taylor: ,What's wrong with negative liberty“, in: A. Ryan (ed.): The idea of freedom, Oxford 1979, 175-195, dt. in:(ders.:) Negative Freiheit?, Frankfurt 1988, 118-144. - Ähnlich wie Taylor argumentieren auch T. Baldwin: ,MacCallum und die zwei Freiheitsbegriffe“, in: Ratio 26 (1984) 105-120, sowie E. Tugendhat: Vorlesungen über Ethik, Frankfurt 1993, $359 f$.

4. Aristoteles: Nikomachische Ethik III, 1-3; V, 10. 
des Wissens und der Abwesenheit von Zwang als solche Ausführungsumstände interpretieren. Allerdings muß er sie damit aus ihrem Kontext lösen. Denn man darf nicht aus den Augen verlieren, daß Aristoteles auch die Bedingung einführt, daß ,freiwillige“ Handlungen ,,bei uns stehen“ müssen, und dieses Kriterium ist unzweifelhaft ein modales. Die Rede davon nämlich, daß eine Handlung ,,bei uns steht“, beinhaltet offenbar zweierlei: (1) daß sie eintreten kann oder nicht und (2) daß die Entscheidung darüber beim Träger der Handlung liegt. Aristoteles und die von ihm beeinflußte Tradition haben an diesen Kriterien festgehalten, so daß man sich auf sie jedenfalls nicht berufen kann, wenn man den modalen Freiheitsbegriff verabschiedet.

Müssen wir aber der Tradition in dieser Beziehung folgen? Müssen wir annehmen, daß nur derjenige prospektiv frei ist, der mehrere Handlungsmöglichkeiten besitzt und selbst darüber entscheidet, welche von ihnen wirklich wird? Und umgekehrt: können wir unter den schon geschehenen Handlungen nur diejenigen retrospektiv als frei bezeichnen, von denen sich zugleich sagen läßt, daß der Handelnde bis zu ihrer Verwirklichung auch anders hätte handeln können? Die stoische Philosophie hat das verneint, ebenso diverse Nachfolger, wie in jüngerer Zeit z.B. Donald Davidson. ${ }^{5}$ Ihnen zufolge kommt es nicht darauf an, daß man verschiedene Alternativen hat, sondern daß die verwirklichten nicht im Widerspruch zu den eigenen Wünschen und Strebungen stehen. Dinge, die man nicht ändern kann, muß man, um frei zu sein, nur nicht mehr ändern wollen. Der stoisch freie Geist zerbricht sich also weder über verschüttete Milch den Kopf, noch darüber, daß er wird sterben müssen oder die gegenwärtigen Qualen ertragen muß, die ihm ein Folterer zufügt. Nun, es ist klar, daß die wenigsten Menschen dies als Freiheit begreifen werden oder auch nur in der Lage sind, eine stoische Haltung einzunehmen. Und selbst wenn sie es durchweg könnten, würde der modale Sinn des Freiheitsbegriffs dadurch nicht widerlegt, sondern eher bestätigt. Denn die Einnahme jener empfohlenen Geisteshaltung muß den Betreffenden, soll die Empfehlung wirksam werden, als Alternative offenstehen. Gewitzte

5. D. Davidson: „Freedom to act“, [orig. 1973] repr. in: (ders.:) Essays on action and events, Oxford 1980, $74 f$. 
Stoiker, Epiktet genauso wie Davidson, gründen deshalb ihre Behauptung, $\mathrm{da} ß$ wir auch gegenüber dem Unabänderlichen frei sein können, nicht auf das schlichte Faktum der Übereinstimmung von Wille und Wirklichkeit, sondern darauf, daß eine Wahlmöglichkeit uns in jedem Fall bleibt, sc. diejenige zwischen dem willigen oder widerwilligen Mitvollzug dessen, was wir als unabwendbar erkannt haben. Der Fuchs, dem die Trauben zu hoch hängen und der sie deshalb nicht mehr will, ist, als verhinderter Traubenesser, zweifellos unfrei, als Lebenskünstler jedoch auch stoisch frei, vorausgesetzt jedenfalls, daß es bei ihm steht, seine Eßwünsche auf die Realitäten abzustimmen.

Was für die fabelhafte Freiheit des Fuchses gilt, gilt für die „positive Freiheit" Charles Taylors ebenso wie für alle Freiheitsbegriffe, die sich prima facie nicht als modale darstellen. Freiheitsbegriffe sind auch sie nur, soweit sie, implizit oder explizit, die Rede von einem Können rechtfertigen. $\mathrm{Da} B$ der liberalistische Nachtwächterstaat kein wirklich freier Staat ist, liegt nicht daran, daß die politische Freiheit, anders als die private, nicht im Besitz von Handlungsalternativen besteht, sondern daran, da $B$ der $Z$ wang durch Gesetze und illegitime staatliche oder private Übergriffe nur einen Teil der Bedingungen ausmacht, die den sozialen Handlungsspielraum begrenzen, so daß ihr Fehlen für die tatsächliche Größe des Spielraums wenig besagen muß. Entsprechendes gilt für jene Aristotelischen Freiheitskriterien, die auf den ersten Blick keine modalen zu sein scheinen. Das Kriterium des Wissens erfüllt seine Funktion nur, weil die vorhandene oder fehlende Kenntnis ebenfalls für die Größe des Handlungsspielraums bedeutsam ist. Wer bestimmte Optionen nicht kennt, die ihm objektiv offenstehen, kann sie ebensowenig nutzen wie diejenigen, die ihm bekannt, aber aus anderen Gründen verschlossen sind. Und die Kriterien der Abwesenheit von äußerem Zwang und des Handlungsbeginns im Inneren sind ebenfalls nicht als solche freiheitsrelevant, sondern nur, weil sie notwendige (wenn auch nicht hinreichende) Bedingungen dafür bilden, daß der Träger der Handlung als derjenige gelten kann, der über die Realisierung bestimmter Optionen entscheidet.

Ich ziehe deshalb den Schluß, daß der Freiheitsbegriff im Kern kein Wirklichkeitsbegriff ist, sondern ein Möglichkeitsbegriff, und daß beide Bedingungen zu seinem modalen Sinn gehören, die in der 
Aristotelischen Rede vom „Bei-uns-Stehen“enthalten sind: (1) die Möglichkeit, so oder anders zu handeln, und (2) die Abhängigkeit der Handlung von uns. Die erste dieser Bedingungen werde ich im folgenden als das Kriterium des ,praktischen Könnens" bezeichnen, die zweite als das der ,Urheberschaft".

\section{Das Problem des freiheitsrelevanten Könnens}

Damit wende ich mich direkt der Frage zu, welcher Sinn von „,Können“ für den Freiheitsbegriff relevant ist. Verdeutlichen wir uns zunächst die Wichtigkeit seiner Spezifizierung. Ein philosophischer Spaßvogel z.B. könnte wie folgt für die Freiheit jeder menschlichen Handlung argumentieren. Jede vollzogene Handlung ist wirklich. Nun gilt aber „Ab esse ad posse valet consequentia“. Folglich sind alle Handlungen frei; denn da der Handelnde sie ausgeführt hat, muß er sie allemal auch haben ausführen können. Die Rabulistik des Arguments liegt nicht nur darin, daß es unserem Kriterium des praktischen Könnens nicht Rechnung trägt und das der Urheberschaft vollständig außer Betracht läßt. Rabulistisch ist es vor allem deshalb, weil es den Sinn des Könnens nicht spezifiziert und den Leser dadurch aufs Glatteis führt. Ist es allein die logische (widerspruchsfreie) Möglichkeit einer Handlung, die durch ihre Wirklichkeit unter Beweis gestellt wird? Ist es ihre naturgesetzliche oder technische Möglichkeit? Oder die situative Fähigkeit oder Gelegenheit des Handelnden zu ihr? Oder will unser Spaßvogel sogar das Palmström-Prinzip umkehren und aus der Wirklichkeit einer Handlung ,,messerscharf" auf ihre Erlaubtheit schließen?6 Ohne spezifizierten Begriff des Könnens ist jeder Freiheitsbeweis gegenstandslos. Er befriedigt uns nicht nur nicht, sondern gibt überhaupt keine Antwort auf unsere Fragen. ${ }^{7}$

6. Ch. Morgenstern: „Die unmögliche Tatsache“, in: (ders.:) Palmström, Berlin 1910.

7. M.R. Ayers (The refutation of determinism, London 1968, 123ff.) scheint dem Spaßvogel-Argument nahezukommen, wenn er die konditionale Analyse des praktischen Könnens mit der Erklärung zu widerlegen versucht, daß ,,no kind of possibility-statement can conceivably be analysed into hypotheticals like those suggested, simply because [..] $p$ entails that $p$ is possible, whereas $p$ does not entail 
Ähnliche Einsichten lassen sich aber auch aus seriöseren Argumenten ableiten. Der Streit zwischen „,Kompatibilisten“ und „Inkompatibilisten" in der Frage der Vereinbarkeit von Freiheit und Determinismus bietet dafür genügend Beispiele. ${ }^{8}$ Richard Taylor und Peter van Inwagen etwa haben einander verwandte (von Aristoteles' klassischer Dartellung in De Interpretatione 9 angeregte) Argumente entwickelt, durch die Taylor den Fatalismus, van Inwagen den „Inkompatibilismus“ und die extreme Seltenheit freier Handlungen beweisen will. ${ }^{9}$ Beide Argumente sind formal korrekt und stützen sich im Kern auf die prima facie ebenso plausible Prämisse, daß man Dinge, die ein für allemal festgelegt sind, nicht ändern kann, und daß dazu auch die Naturgesetze und die vergangenen Tatsachen gehören. Dennoch haben beide Autoren ihre Gegner nicht überzeugt. Warum? Die Diskussion, die vor allem bei Taylor recht ausgedehnt war, hat sich zu einem erheblichen Teil auf Nebenkriegsschauplätzen bewegt. Der zentrale Kontroverspunkt ist jedoch nicht, ob die Annahmen einer fixierten Vergangenheit oder einer Fixierung (nicht nur Probabilisierung) präsenter durch fixierte vergangene Weltzustände überhaupt sinnvoll sind, oder wenn ja, ob man die Möglichkeit, anders zu handeln, durch die mangelnde Rigidität der Kausalgesetze oder vielmehr (mit oder ohne Rekurs auf ,,Rückwärtskausalität“) durch die Änderbarkeit der Vergangenheit verständlich macht. ${ }^{10}$ Und der Kern des Streits liegt auch nicht

that if $q$, then $p$ " (a.a.O.,128). So einfach aber läßt sich die Analyse gewiß nicht verabschieden.

8. Ich setze die Ausdrücke in Anführungszeichen, weil die Rede von Befürwortern oder Gegnern ein und derselben ,,Vereinbarkeitsthese“ nach meiner Meinung den Kern des Streites verdeckt; vgl. G. Seebaß: Freiheit und Determinismus, in: Zeitschrift für philosophische Forschung 47 (1993), $14 \mathrm{ff.}$

9. R. Taylor: „Fatalism“, in: The Philosophical Review 71 (1962), 56-66; ders.: Metaphysics, Englewood Cliffs 1963, 54-69; P.v. Inwagen: „The incompatibility of free will and determinism“, in: Philosophical studies 27 (1975), 185-199, dt. in: U. Pothast (ed.): Freies Handeln und Determinismus, Frankfurt 1978, 247-265; ders.: An essay on free will, Oxford 1983; ders.: ,When is the will free?", in: Philosophical perspectives 3 (1989), 399-422.

10. Für die zuletzt genannten Alternativen vgl. insbesondere D. Lewis: ,Are we free to break the laws?", in: Theoria 47 (1981), 113-121 und J. Bennett: „Counterfactuals and temporal direction", in: The philosophical review 93 (1984), 57-91, sowie zu beiden C. Ginet: On action, Cambridge 1990, 107ff. Kritik an der 
darin, ob man berechtigt ist, von der gesicherten Unbeeinflußbarkeit vergangener Sachverhalte auf die Unbeeinflußbarkeit künftiger, durch sie fixierter Sachverhalte zu schließen. ${ }^{11}$ Entscheidend ist nur die Deutung des Könnens. ${ }^{12} \mathrm{Daß}$ man in einem gewissen Sinn davon

ontologischen Asymmetrie der Zeit und der Existenz hinreichender Kausalbedingungen haben in der Diskussion von Taylors Argument eine Rolle gespielt; vgl. z.B. L. Spellman: ,Forthcoming sea fights: what theory of time?“", in: The new scholasticism 55 (1981), 61ff., und J.E. Tomberlin: ,A fatalistic paradox examined", in: Philosophy and phenomenological research 39 (1979), 591.

11. Vgl. dazu vor allem die Diskussion zwischen P. v. Inwagen 1989, a.a.O.; J.M. Fischer/M. Ravizza: ,When the will is free“, in: Philosophical perspectives 6 (1992), 423-451 und P. v. Inwagen: ,When the will is not free", in: Philosophical studies 75 (1994), 95-113. Zwar werden ,,Kompatibilisten“ das Schlußprinzip, das van Inwagen (1989, a.a.O., 405) als das zentrale Merkmal des ,,Inkompatibilismus“ hinstellt, in aller Regel bestreiten. Doch sie müssen es nicht unbedingt, und wenn sie es tun, liegt der Grund nicht in der vermeintlichen logischen Ungültigkeit des Prinzips, sondern in einem anders gefaßten Begriff des praktischen Könnens, speziell des konditionalen.

12. Daß hier der zentrale Streitpunkt liegt, ist in der Diskussion oft nicht deutlich geworden. Nur indirekt haben jene Kritiker Taylors auf ihn verwiesen, die den (vergeblichen) Versuch unternahmen, sein Fatalismus-Argument mit dem Hinweis auf die Differenz zwischen kausaler und logischer Notwendigkeit bzw. auf den Fähigkeits-Sinn von ,können“" (vgl. dazu S. 222ff.) zu widerlegen, vgl. z.B. B. Aune: „Fatalism and professor Taylor“, in: The philosophical review 71 (1962), $513 \mathrm{ff}$.; R. Abelson: ,Taylor's fatal fallacy“, in: The philosophical review 7 (1963), 94ff., und R. Segal: ,Fatalism“, in: Pacific philosophical quarterly 62 (1981), $371 \mathrm{ff}$. Van Inwagen wiederum hat ihn als Kontroverspunkt zwischen ihm und dem konditionalanalytischen ,,Kompatibilismus“ anerkannt, ihn aber zusammen mit diesem relativ rasch vom Tisch gewischt (1975, a.a.O., 261ff.) und die Frage des Sinns von ,können“" später sogar für gänzlich irrelevant erklärt, da alle Seiten gemeinsam ,,den Alltagssinn“ zugrundelegen würden (1989, a.a.O., 403f.).

Demgegenüber hatte schon G.E. Moore (vgl. Anm. 14) seinen konditionalen, „,kompatibilistischen“ Begriff des Anderskönnens nicht als den einzig sinnvollen aufgefaßt, sondern ihn neben einen von ihm nicht angezweifelten ,, inkompatibilistischen" Begriff gestellt. Der Verlust dieser Unterscheidung hat später dazu geführt, daß oft aneinander vorbeigeredet wurde. Für die Diskussion etwa, die sich an Nelson Pikes vielbeachtetes Argument für die Unvereinbarkeit von Freiheit und Allwissenheit anschloß, hat W.P. Alston dies detailliert nachgewiesen; vgl. ,Divine foreknowledge and alternative conceptions of human freedom“, [orig. 1985] repr. in: J.M. Fischer (ed.): God, foreknowledge, and freedom, Stanford 1989, 258-273. 
sprechen kann, Dinge nicht ändern zu können, die ein für allemal festliegen, einschließlich der Vergangenheit und der Naturgesetze und einiger durch sie fixierter zukünftiger Ereignisse, müssen und wollen die ,kompatibilistischen“ Gegner Taylors und van Inwagens normalerweise nicht bestreiten. Sie bestreiten jedoch, daß dies der Sinn ist, der für den Freiheitsbegriff Bedeutung hat. Das mag seine Richtigkeit haben oder auch nicht. Entscheiden läßt sich die Kontroverse jedenfalls erst, wenn beide Seiten sich nicht länger darauf beschränken, der Gegenposition eine korrekt formulierte, aber der Sache nach falsche Behauptung zu attestieren, sondern den Sinn der Rede vom Können als Kern ihres Streits begreifen und den jeweils für richtig gehaltenen spezifizieren. Solange dieser Schritt nicht getan ist, ist jeder Streit um die Vereinbarkeit von Freiheit und Determinismus witzlos.

\section{Die konditionale Analyse}

Was aber ist der gesuchte Sinn? Die traditionelle Antwort der Theoretiker, die zur Gruppe der ,,Kompatibilisten“ gehören, ist die konditionale Analyse des praktischen Könnens. Sie ist auf Individuen gemünzt, die wollen und willentlich handeln können, und besagt im Kern, daß sich die kategorische Aussage, daß jemand so oder anders handeln kann, auf die hypothetische Aussage zurückführen läßt, daß er handeln wird, wenn er es will, und nicht handeln, wenn er es nicht will. Schematisch läßt sich das so zusammenfassen: ${ }^{13}$

13. Ich gehe hier zunächst vom expliziten Wollen einer Handlung oder ihres Gegenteils aus und stelle die Willensindifferenz bis zum nächsten Abschnitt (S. 213ff.) zurück. Dabei kann offen bleiben, ob der gewollte Sachverhalt direkt vom Willen abhängt (Aufstehen oder Sitzenbleiben) oder nur indirekt über diverse Handlungsfolgen (telefonische Hotelbuchung in Australien). Ausklammern muß ich hier allerdings diejenige Rede vom ,,Können“, die sich auf Handlungen oder Handlungsfolgen bezieht, für die der präsente Wille nicht hinreicht (,,Peter kann in zwei Stunden in London sein“, ,,Anke kann eine Spitzenspielerin werden“). Vertreter der Konditionalanalyse müssen behaupten, daß diese komplexeren Fälle sich schrittweise auf Fälle der präsenten Willensabhängigkeit zurückführen lassen. Obwohl das nicht selbstverständlich ist, werde ich diesen Punkt im folgenden schenken. 
(K) Ein beliebiges Individuum $\mathrm{x}$ ist einem beliebigen Handlungspaar $\mathrm{h} / \mathrm{h}$ gegenüber genau dann frei, d.h. kann $\mathrm{h}$ oder - $\mathrm{h}$ ausführen, wenn unter den bestehenden Restbedingungen folgende Konditionalsätze wahr sind:

(1) wenn $x$ will, daß $h$, dann $h$, und

(2) wenn $x$ will, daß -h, dann - $h$.

Da beide Alternativen, das Tun wie das Unterlassen von $\mathrm{h}$, relativ auf das sie bedingende Wollen, offen sind, scheint unser Kriterium des praktischen Könnens erfüllt. Und da die kombinierten Konditionalsätze zum Ausdruck bringen, daß der Wille des Handelnden über den Eintritt von h oder -h entscheidet, wird insoweit offenbar auch dem Kriterium der Urheberschaft Rechnung getragen.

Die konditionale Analyse des praktischen Könnens wird in der Analytischen Philosophie gewöhnlich auf Moore zurückgeführt, dessen einschlägige Darstellung von Austin kritisiert und von neueren Autoren zumeist verteidigt wurde. ${ }^{14}$ Doch sie ist keine neue Erfindung. Auch ihre analytischen Anhänger wissen das, glauben im allgemeinen jedoch, ihre Wurzeln im britischen Empirismus festmachen zu können, insbesondere bei Hobbes und Hume. Diese Diagnose greift historisch wie systematisch zu kurz. Wir finden die Konditionalanalyse auch bei neuzeitlichen Philosophen wie Leibniz und Schopenhauer, ${ }^{15}$ die gewiß keine Empiristen sind, und ihr historischer Ursprung liegt allemal wesentlich früher und an einer ganz anderen Stelle. Shakespeare scheint sie gekannt zu haben, zumindest in einer Lesart des kurzen Dramendialogs, den ich die-

14. Vgl. G.E. Moore: Ethics, [orig. 1912] repr. Oxford 1965, ch. VI; (ders.:) „Replies: 8. Freedom of choice“, in: P.A. Schilpp (ed.): The philosophy of G.E. Moore, Cambridge 1942, 623-627, und J.L. Austin: „Ifs and cans“, [orig. 1956] repr. in: (ders.) Philosophical papers, Oxford 1970, 205-232. Zu den Nachfolgern oder Verteidigern Moores gehören (u.a.) C.L. Stevenson: Ethics and language, New Haven 1944, ch. XIV; P.H. Nowell-Smith: Ethics, Harmondsworth 1954, 273ff.; A.I. Goldman: A theory of human action, Englewood Cliffs 1970, 64; Davidson 1973, a.a.O., 66ff., und E. Tugendhat: „Der Begriff der Willensfreiheit“, in: K. Cramer et al. (eds.): Theorie der Subjektivität, Frankfurt 1987, 383ff.

15. Vgl. Leibniz: Von der Allmacht und Allwissenheit Gottes und der Freiheit des Menschen, Akademie-Ausgabe Bd. VI/1, 541; Schopenhauer: Preisschrift über die Freiheit des Willens, Zürcher Ausgabe (ed. A.Hübscher) Bd. VI, Zürich 1977, 56f., 82. 
sem Aufsatz als Motto vorangestellt habe. ${ }^{16}$ Ursprünglich entwickelt aber wurde sie nicht in der Philosophie, sondern in einem außerphilosophischen, ganz und gar nichtempiristischen Kontext. Gemeint ist die (christliche) Theologie, wo die konditionale Analyse seit langem eingeführt ist und die formal gleiche Funktion erfüllt wie in späteren Theorien.

Die Theologie hat bekanntlich die Schwierigkeit, die These von der Determiniertheit alles Geschehens, die sich aus der Allwissenheit und Allmacht Gottes ergibt, mit der menschlichen Freiheit in Einklang zu bringen. Menschen, die nicht anders handeln können, als sie es faktisch und (ex hypothesi) göttlich vorherbestimmt tun, lassen sich schwerlich für ihre Taten verantwortlich machen. Gott also, wenn er sie dafür tadelt, erscheint nicht nur ungerecht, sondern als Urheber dieses Geschehens auch scheinheilig. Die konditionale Analyse eröffnet den Ausweg aus diesem Dilemma. Sie macht es möglich, von einem bedingten Anderskönnen auch dann zu reden, wenn der Nichteintritt der Bedingung feststeht. In diesem Sinne hat Anselm von Canterbury z.B. erklärt, kategorische Sätze der Form , $\mathrm{X}$ kann“ hätten in derjenigen Verwendung, in der sie $\mathrm{x}$ eine ,Macht" (,,potestas") zuschreiben, vollständig expliziert die hypothetische Form ,, $\mathrm{x}$ kann, wenn $\mathrm{x}$ will“, wobei nicht vorausgesetzt sei, daß auch das Antecedens, das Wollen von $\mathrm{x}$, anders ausfallen könnte. ${ }^{17}$ Der Stammvater dieses Gedankens ist Augustin. Er hat den neuzeitlichen Begriff der Handlungsfreiheit antizipiert und die konditionale Analyse eingeführt in dem Bestreben, beiden Bedingungen, die in der Aristotelischen Rede vom ,,Bei-uns-Stehen“ (,,eph' hemin“) freier Handlungen enthalten sind, gleichzeitig Rechnung zu tragen, sc. der des praktischen Könnens und der der Urheberschaft (S. 207). Grundlage dafür ist eine spezielle Definition von ,,in unserer Macht stehen“ bzw. ,,frei sein“ und „,können“. Ihr zufolge steht ein beliebiger Sachverhalt p, der eine Handlung oder Handlungsfolge beschreibt, genau dann nicht in unserer Macht (,,in potestate nostra"), wenn einer der folgenden Fälle auftritt: entweder wir wollen $\mathrm{p}$ und faktisch $-\mathrm{p}$ oder wir wollen $-\mathrm{p}$ und faktisch $\mathrm{p} .^{18}$

16. Auf eine zweite Lesart komme ich am Ende des Aufsatzes (S. 227) zurück.

17. Anselm v. Canterbury: Cur deus homo II,1.

18. Augustin: De libero arbitrio III, 14-41; De civitate dei V, 9-10. 
Formt man die negative Formulierung Augustins positiv um und ersetzt die sich ergebende negierte Konjunktion durch die materiale Implikation, gewinnt man die konditionale Analyse.

Ich bin auf diese Hintergründe natürlich nicht ohne Hintergedanken zu sprechen gekommen. Die konditionale Analyse entspringt dem Interesse, zwei Dinge miteinander in Einklang zu bringen, die offenbar nicht zu vereinbaren sind. Neuere Philosophen, die mit ihr sympathisieren, werden vermutlich gern bereit sein, den auf sie gegründeten theologischen „Kompatibilismus“ als einen intellektuellen salto mortale zu begreifen, der von Wunschdenken mehr als von Einsicht getragen ist. Wenn der Augustinische Ansatz jedoch verfehlt ist, ist er es nicht nur hier, sondern überall. Wer die von religiösen Vorgaben diktierten Versuche zur Lösung des Theodizeeproblems als Ausflucht belächelt, sollte sich eingestehen, daß auch ihre säkularen Analoga, wie sie von Hume oder Moore entwickelt wurden, ,kompatibilistisches“ Wunschdenken sind. Ich möchte daraus die Konsequenzen ziehen und im folgenden versuchen, die Schwachpunkte der Konditionalanalyse, frei von theologischen und philosophischen Vorgaben, herauszuarbeiten. Dabei werde ich mich, was das willensbedingte Handeln angeht, der Einfachheit halber auf elementare Körperbewegungen beschränken, wie z.B. das Heben des rechten Arms, und zugleich voraussetzen, daß die Konditionale kausal und deterministisch zu interpretieren sind, also nicht logisch oder probabilistisch. Die entscheidenden Punkte werden von dieser Vereinfachung nicht berührt. Und daß auch elementare Körperbewegungen keineswegs nur triviale Handlungsalternativen bieten, kann man sich leicht daran klar machen, daß das Heben des rechten Arms in einer Abstimmung über Krieg oder Frieden entscheiden kann.

\section{Der Einwand der Willensfreiheit}

Ein Einwand gegen die konditionale Analyse geht dahin, daß sie nicht alle Alternativen erfaßt und die erfaßten zu weit von der Wirklichkeit abkoppelt. Das Handlungspaar h/-h ist eine logisch vollständige Disjunktion. Auf der Bedingungsebene aber gibt es nicht nur das Wollen von $\mathrm{h}$ und das Wollen von $-\mathrm{h}$, sondern auch 
die Willensindifferenz, also die Abwesenheit jeder Stellungnahme zur kritischen Alternative. Die konditionale Analyse verlangt nur, $\mathrm{daß} \mathrm{h}$ oder - $\mathrm{h}$ eintritt, wenn der Handelnde das relevante Wollen entwickelt. Tut er das nicht, sind ihre Bedingungen trivialerweise erfüllt. Folglich könnte ein logisch gewitzter, phantasievoller, ,Konditionalist" den Beweis dafür antreten, daß auch ein Stein in labiler Gleichgewichtslage frei ist, zu fallen oder liegen zu bleiben. Denn wenn er den fraglichen Willen hätte, würde er sich, gegeben geeignete Restbedingungen, nach unten bewegen. Nur will er es eben nicht und kann es nicht wollen. (Wer Schwierigkeiten hat, sich einen Stein in dieser Lage zu denken, mag einen Schachcomputer oder die jeweils beste Maschine, die die KI als Analogon handlungsfähiger Menschen anbietet, an seine Stelle setzen). Nun werden sich manche hier vielleicht an Wittgensteins Diktum erinnern, daß es Unsinn sei zu sagen, ,,der Sessel denke bei sich“" oder ähnliches, und von daher geneigt sein, den Stein und alle Objekte, die sich nicht gerade wie willensfähige Menschen benehmen, kategorial auszuschließen. ${ }^{19}$ Ohne Voraussetzung eines (in meinen Augen: unhaltbaren) behavioralen Funktionalismus ist dieser Ausschluß kaum zu rechtfertigen. Doch unterstellen wir einmal, daß er begründet ist. Was wäre damit gewonnen? In der entscheidenden Hinsicht nichts. Auch willensfähige Menschen müssen ja nicht zu allen Optionen, die sie besitzen, volitiv Stellung nehmen oder sich ihrer auch nur bewußt sein. Wenn sie es nicht sind, sind die Kriterien des konditionalen Könnens ebenfalls trivial erfüllt. Sollen wir sie deshalb für frei erklären? Dann hätte auch der Obdachlose, der jetzt von Almosen leben muß, die Freiheit gehabt, ein Millionär zu sein, da er unzweifelhaft einer geworden wäre, hätte er nur letzte Woche die Zahlenreihe mit Willen getippt, auf die der Haupttreffer im Lotto fiel. Wenn wir Zynismen dieser Art ausschließen wollen, können wir uns mit der bloßen Konditionalanalyse nicht begnügen.

Offensichtlich ist es nicht gleichgültig, in welchem epistemischen

19. Vgl. L. Wittgenstein: Philosophische Untersuchungen, Oxford 1958, § 361. Stevenson (a.a.O., 299 note) wollte weniger weit gehen und die Konditionalanalyse nur mit dem Argument verteidigen, daß die Naturgesetze ihre Anwendbarkeit auf Pflanzen und sonstige, insensible objects“ ausschlössen, daß sie jedoch nicht sinnlos sei. Das ist plausibler als der Wittgensteinianische Rigorismus, bestätigt aber das explikative Defizit. 
und volitiven Zustand sich das mutmaßlich freie Individuum befindet und wie es in ihn hinein geriet. Beschränken wir uns hier einmal auf das Wollen allein und betrachten nur die bewußte Stellungnahme zu einem einzigen Handlungspaar $\mathrm{h} / \mathrm{h}$. Trotz dieser weiteren Simplifizierung der explikativen Aufgabe ist das Ungenügen der Konditionalanalyse offenkundig. Auch Triebtäter, Drogensüchtige, Gefolterte oder Erpreßte wollen ja, was sie wollen, bewußt. Dennoch sind sie ihm gegenüber nicht frei. Der freie Mensch, so scheint es, muß nicht nur anders handeln können, sondern auch anders wollen. Das ist der klassische Einwand der Willensfreiheit. ${ }^{20}$ Anhänger der Konditionalanalyse haben sich ihm z.T. zu entziehen versucht, indem sie die Notwendigkeit der Frage nach der Freiheit des Wollens bestritten oder zu zeigen versuchten, daß schon der Begriff der „Willensfreiheit" ein Unding sei. ${ }^{21}$ Beides ist nicht überzeugend. Ausweichen kann man der Frage allenfalls, wenn man noch einen anderen Gedanken der Augustinischen Tradition positiv aufgreift. Augustin nämlich hielt seine Analyse deshalb für hinreichend, weil er beweisen zu können glaubte, daß das Wollen per se als ,,frei“ zu betrachten ist. Nach seiner Darstellung ist es begrifflich ausgeschlossen, daß die Leistung des „Wollens“ selbst die Kriterien des willensabhängigen ,,In-unserer-Macht-Stehens" (S. 212f.) nicht erfüllt. ${ }^{22}$ Das Argument scheitert formal, da Augustin an dieser Stelle

20. Auch in der Kontroverse um Moores Analyse und Austins Kritik hat er eine Rolle gespielt. Roderick Chisholm (, J.L. Austin's Philosophical Papers“, in: Mind 73, 1964, 24f.) hat ihn als die entscheidende Schwäche jeder konditionalen Analyse betrachtet und A.C. Garnett hat seine Kritik an Moore darauf aufgebaut (,Moore's theory of moral freedom", in Schilpp (ed.) 1942, a.a.O., 179-199). Moore selbst war sich des Problems zwar von Beginn an bewußt (1912, a.a.O., 94f.), glaubte sich seiner jedoch im Rahmen der Konditionalanalyse durch die Einführung der „Fähigkeit, anders wählen zu können“ als schlichte Zusatzbedingung entledigen zu können (1942, a.a.O., 624).

21. Das bedeutendste Argument hierfür ist der Regreß-Einwand, der seit Hobbes (Works, ed. Molesworth, IV, 69 u.a.) von zahllosen Philosophen erhoben wird. Drei weitere Argumente neben dem Regreßargument finden sich bei Locke (Essay II, 21,14-16.23-25.48). Ich halte all diese Widerlegungsversuche für gescheitert, muß hier aus naheliegenden Gründen jedoch auf ihre detaillierte Kritik verzichten.

22. Vgl. Anm. 18 und zur Kritik W. Rowe:,,Augustine on foreknowledge and free will", in: The review of metaphysics 18 (1964), 356-363. 
das höherstufige Wollen nicht berücksichtigt, das er anderswo sehr wohl in Rechnung stellt und (wie neuerlich auch Moore und vor allem Harry Frankfurt) auf das Problem der Willensfreiheit bezieht. ${ }^{23}$ Die Übertragung der Konditionalanalyse von der Stufe des Handelns auf die des Wollens (erster oder beliebiger höherer Stufen) ist, wenn Augustins Beweis wegfällt, die einzige Möglichkeit, im Rahmen dieses Konzepts Sinn mit dem Problem der Willensfreiheit zu machen. Eine wirkliche Lösung enthält dieser Schritt aber nicht, da die Frage des konditionalen Könnens natürlich auf jeder höheren Stufe wiederkehrt.

Ich belasse es bei diesem kurzen Blick auf das Problem der Willensfreiheit und das generelle Problem des Zustandekommens derjenigen volitiven und epistemischen Zustände, die unser Handeln faktisch bestimmen. ${ }^{24}$ Mich interessieren hier nur die kritischen Konsequenzen für die Konditionalanalyse. Diese hat sich in zweierlei Hinsicht als unzureichend erwiesen. Als Explikation des freiheitsrelevanten Sinnes von ,,können“ ist sie einerseits unvollständig, da sie, spätestens auf einer relevanten höheren Stufe des Wollens, der Ergänzung durch andere Freiheitskriterien bedarf. Die Frage des praktischen Könnens wird durch sie also nicht hinreichend beantwortet. Zugleich aber ist sie prinzipiell ungeeignet, der Bedingung der Urheberschaft Genüge zu tun. Denn ohne den weiterreichenden Beweisanspruch Augustins, wonach jedes Wollen ,,in unserer Macht steht“", ist nicht zu sehen, warum Willenszustände bzw. deren mentale oder physische Antezedentien in dieser Hinsicht ausgezeichnet sein sollten. Beiden Bedingungen also, die in der Aristotelischen Rede vom „Bei-uns-Stehen“ enthalten sind, wird die konditionale Explikation nicht gerecht.

23. Vgl. Augustin: Confessiones VIII, 5,10ff.; Retractationes XII,5; Moore 1912, a.a.O., 93-95; H. Frankfurt: „Freedom of the will and the concept of a person", in: The journal of philosophy 68 (1971), 5-20.

24. An anderer Stelle habe ich ausführlicher dazu Stellung genommen; vgl. Seebaß 1993, a.a.O. (Anm.1), Kap. VI, 3-4. 


\section{Modale Signifikanz und modale Spezifizierung}

Nun könnten ihre Verteidiger allerdings geltend machen, daß dies auch eine falsche Erwartung sei. Die konditionale Analyse könne zwar keine Explikation des Freiheitsbegriffs und des freiheitsrelevanten Könnens im ganzen geben, wohl aber sei sie geeignet, den Sinn von „Können“ offenzulegen, der für das freie Handeln charakteristisch ist. Ob der Abgeordnete Schulz, der als Hinterbänkler den Wahlkreis Hanau vertritt, auch darin frei war, daß er den Willen entwickelte, den Arm zu heben und das Anti-Atomgesetz damit scheitern zu lassen, mag zweifelhaft sein und von diversen Zusatzbedingungen abhängen. Doch daß er frei war, anders zu handeln, steht fest, vorausgesetzt nur, daß sein Arm seinem Willen gehorchte. Können wir demnach sagen, daß die konditionale Analyse den modalen Sinn, wenn nicht der Willensfreiheit, so doch der Handlungsfreiheit erfaßt?

Ich möchte zeigen, daß sie auch diese Erwartung enttäuscht. Nach der konditionalen Analyse soll der Satz ,x kann h/-h" soviel bedeuten wie ,, wenn $x$ will, daß $h$, dann $h$, und wenn $x$ will, daß -h, dann - $h$ ". So einander gegenübergestellt zeigen beide Sätze jedoch eine auffällige formale Disanalogie: das Explikandum ist modalisiert, daß Explikans nicht. Das muß Verdacht wecken. Könnte es sein, daß das praktische Können hier weniger expliziert als eliminiert werden soll? Extensionalisten wird dies willkommen sein. Doch stellt sich auch hier, wie bei allen Reduktionen, die Frage, was dabei unter den Tisch fällt. Zwei Minimalbedingungen sollten erfüllt werden. Um überhaupt als Explikation eines Modalausdrucks in Frage zu kommen, sollte die Analyse modal signifikant sein, d.h. zu erkennen geben, daß die Pointe der modalen Rede getroffen ist, auch wenn sie selbst nicht mehr modal sein sollte. Zusätzlich aber und vor allem muß sie den Sinn von „Können“ spezifizieren, um ihrem explikativen Anspruch Genüge zu tun.

Prüfen wir zunächst die Signifikanzbedingung. Worin kann sie bestehen? Nach dem zu Anfang Gesagten liegt es nahe, sich am modalen Gattungsbegriff zu orientieren, den Aristoteles formuliert hat. Danach muß jedem Möglichkeitsbegriff, also auch jedem explikativen Substitut für einen solchen, ein Spielraum zugeordnet sein, in dem mehrere Alternativen als offen gelten. Ist das in der 
Konditionalanalyse der Fall? Es scheint so. Denn die beiden Konditionale beschreiben ja, angewandt auf ein einzelnes Handlungspaar h/-h, zwei relevante Möglichkeiten, ohne sich auf eine festzulegen. Allerdings tun sie das nicht in modalisierter Form. Sie bilden zwar eine Konjunktion, in der beide Konjunkte faktisch einander korrespondieren, aber sie sagen nicht, $d a \beta$ dies so ist und daß beide Alternativen offen sind. Vielmehr steht jeder Konditionalsatz zunächst für sich und sagt nur, daß zwei Sachverhalte aneinander

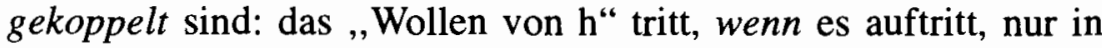
Kombination mit , $h$ " auf, das ,, Wollen von $-h$ " nur in Kombination mit ,,-h".

Daß es sich um ein Satzpaar handelt, das Sachverhalte beschreibt, die korrespondierende Alternativen bilden, $m u \beta$ also nicht modal gedeutet werden. Tut man es aber, ergibt sich zunächst auch nur der Blick auf zwei Segmente denkbarer Weltverläufe, die als Folgen von Wollen und Handeln beschrieben werden, über deren Offenheit aber nichts ausgesagt wird. ${ }^{25}$ Letztere kann gegeben sein oder auch nicht. Nur die Beschreibung als Folge ist es jedoch, für die die konditionale Formulierung verantwortlich zeichnet. Für die modale Deutung ist sie ohne Belang. Beide Alternativen hätten auch undifferenziert, als ein einziger zeitlich erstreckter Vorgang beschrieben werden können. (Etwa so, wie wir undifferenziert von einem ,,Heben des rechten Arms" reden, ohne es in einzelne Phasen zu zerlegen.) Damit aber hat sich das Spezifikum der Konditionalanalyse als explikativ irrelevant erwiesen. Für sich genommen ist die Aussage über die Kausalbedingtheit einer Handlung durchs Wollen ungefähr so informativ wie die Aussage, daß ein denkbarer Lottogewinn nicht

25. Genauer gesagt: die Fälle Wh \& $-\mathrm{h}$ und W-h \& h sind (ex hypothesi) ausgeschlossen, während die restlichen prinzipiell in Betracht kommen. Dabei handelt es sich (wenn widersprüchliche Willenslagen außer Betracht bleiben) um die fokal ins Auge gefaßten Fälle des Willenshandelns Wh \& h und W-h \& -h, sowie um die Indifferenzfälle -Wh \& -W-h \& h und -Wh \& -W-h \& -h. Doch es wird nichts darüber gesagt, ob weitere Restriktionen den verbliebenen Spielraum verengen. Das hat gravierende Konsequenzen. Auf der Basis der schlichten, nicht weiter spezifizierten Konditionalanalyse müßte man z.B. sagen, daß ein Mensch auch dann frei ist, $\mathrm{h}$ zu tun, wenn das willenlose Nichteintreten von $\mathrm{h}(=-\mathrm{Wh} \&$ -W-h \& -h) die einzig offene Alternative und somit (nach dem zu Anfang Gesagten, S. 201f.) notwendig ist. 
als ein einzelner Scheck, sondern als Bündel mehrerer Banknoten zur Auszahlung käme, falls er erzielt wird. Insoweit also ist der Versuch, die modale Signifikanz der Konditionalanalyse unter Beweis zu stellen, gescheitert.

Gibt es sie dann überhaupt? Tatsächlich dürfte wohl niemand auf den Gedanken verfallen sein, den konditional formulierten Zusammenhang zwischen Wollen und Handeln als Explikation des Satzes auszugeben, daß der Träger handeln kann, gäbe es nicht einen Musterfall, der bei diesem Verfahren Pate gestanden hat und dessen modale Signifikanz gesichert erscheint. Gemeint sind gewöhnliche, nicht auf Willenshandlungen, sondern auf Stoffe und materielle Objekte bezogene Dispositionsprädikate. Im Deutschen werden diese zumeist durch Adjektive mit Suffixen wie ,lich“ oder ,,bar“ gebildet. Daß ein Stück Zucker löslich oder eine Fensterscheibe zerbrechlich ist, heißt nichts anderes, als daß die Folge des SichLösens bzw. Zerbrechens eintritt, wenn bestimmte Umstände eintreten. Die Suffixe „bar“ und ,lich“ jedoch sind im Deutschen modal qualifiziert. Statt zu sagen, daß eine Fensterscheibe zerbrechlich ist, kann man auch sagen, daß ihr Zerbrechen möglich ist oder daß sie zerbrechen kann. Ähnliches gilt für andere Sprachen.

Freilich: ist diese modale Wendung, die als sprachliche Tatsache unbestreitbar erscheint, auch sachlich gerechtfertigt? Nach der Auffassung von Hobbes und neuerlich Nelson Goodman ist sie es nicht. ${ }^{26}$ Denn ihr zufolge bezieht sich die Aussage, daß eine (zur Zeit noch unzerbrochene) Fensterscheibe ,, zerbrechlich " ist, nur auf den (unbekannten) späteren Zeitpunkt, zu dem sie wirklich zerbricht. Fehlt ein solcher, d.h. bleibt die Scheibe dauerhaft heil, ist die Aussage falsch oder gegenstandslos. Nach dieser Auffassung also soll sich die Dispositionszuschreibung nicht auf verschiedene, alternative Weltverläufe beziehen, sondern nur auf den einen, der wirklich wird. Doch lassen wir diese Extremposition, die der gewöhnlichen Auffassung nicht entspricht und sichtbar von philosophischen Vorgaben diktiert ist, hier einmal außer Betracht. Normalerweise verstehen wir Dispositionen jedenfalls als Eigenschaften, die ihren Trägern zukommen im Hinblick auf etwas, das mit ihnen

26. Hobbes: De corpore X, 1.5; N. Goodman: Fact, fiction, and forecast, London 1954, $40 \mathrm{ff}$. 
geschehen kann, auch wenn es faktisch nie eintreten sollte, oder das kontrafaktisch mit ihnen hätte geschehen sein können. Würde die Scheibe von diesem Stein, der in ihre Richtung geschleudert wurde, sie aber vielleicht verfehlt, tatsächlich getroffen, würde sie brechen. Wäre das Zuckerstück, das nun unberührt neben ihr liegt, beim Einschenken in der Kaffeetasse gewesen, hätte es sich gelöst. Dispositionelle Konditionale sind kontrafaktische oder rein hypothetische Konditionale, die alternative Weltverläufe in Rechnung stellen. Wenn wir die konditionale Analyse des praktischen Könnens nach diesem Muster verstehen, ist ihre modale Signifikanz gesichert. Sie bezieht sich dann, wie gewöhnliche Dispositionen, auf einen Spielraum von Alternativen, die sie als kontrafaktisch oder antezedent offene hinstellt. Würde Schulz seinen Arm, contra oder ante factum betrachtet, nicht heben wollen, bliebe er unten. Die modale Signifikanzbedingung also kann eine dispositionell interpretierte Konditionalanalyse anscheinend erfüllen.

\section{Kausalgesetzliches Können?}

Wie aber steht es in dieser Hinsicht mit der Bedingung der modalen Spezifizierung? Der Sinn eines Modalausdrucks hängt, wie eingangs festgestellt (S. 201f.), von der Art der Alternativen ab und von den Gründen, die sie als offen oder verschlossen erweisen. Die Art der Alternativen ist beim praktischen Können klar. Es geht um Handlungen, und zwar im einfachsten Fall um kontradiktorische Handlungspaare der Form ,h/-h“. Kritisch allerdings ist die Frage der Gründe. Worauf beziehen wir uns, wenn wir sagen, daß beide Alternativen, das willensabhängige Tun oder Unterlassen von h, offen sind oder vor ihrem Eintritt offen waren?

Orientieren wir uns zunächst wieder am Vorbild gewöhnlicher Dispositionsprädikate. Worauf stützen wir uns, wenn wir sagen, daß das Zerbrechen einer zerbrechlichen, bislang heil gebliebenen Scheibe eine offene Möglichkeit ist oder war? Die Antwort liegt auf der Hand. Beide Alternativen, das Bersten von Glas unter Druck oder Stoß und sein Heilbleiben bei deren Fehlen, gehören zum Kernbestand unseres Kausalwissens; genauer gesagt: unseres Wissens um kausale Gesetzmäßigkeiten, die wir (oder andere) empi- 
risch gewonnen haben und nun induktiv extrapolieren. Auf sie beziehen wir uns, wenn wir beide Alternativen für offen erklären. Besonders deutlich ist das in der kontrafaktischen Rede. Natürlich meinen wir, wenn wir sagen, daß die heil gebliebene Scheibe auch hätte zerbrechen können, nicht, daß diese faktisch nicht realisierte Möglichkeit ex post doch noch real werden könnte. Faktisch ist sie verschlossen. Wenn wir sie kontrafaktisch gleichwohl zu den offenen rechnen, so nur, weil sie zu den Alternativen des theoretischen Spielraums gehört, die durch die kausalen Gesetzmäßigkeiten nicht ausgeschlossen werden. ${ }^{27}$ Dieser Grund für den Ausschluß wird verneint. Der Sinn der Rede vom ,,Können“ also ist hier der naturgesetzliche bzw. kausalgesetzliche in einem weniger anspruchsvollen, alltäglichen Sinne. Die notorischen Schwierigkeiten, die sich ergeben, wenn man ihn präzisieren oder philosophisch tiefer begründen will, können wir hier übergehen. Denn es ist allemal klar, daß dieser Sinn für die Analyse des praktischen Könnens ausscheidet. $\mathrm{Zu}$ sagen, daß jemand deshalb frei ist oder frei war, so oder anders zu handeln, weil beides den Naturgesetzen nicht widerspricht, ist beinahe ebenso lächerlich wie die Leibnitianische Erklä-

27. Es ist zwar, wie vor allem David Lewis immer wieder betont hat, nicht theoretisch selbstverständlich, daß der Spielraum des kontrafaktisch Möglichen durch unveränderliche, generelle Gesetze bestimmt wird, die verschiedene Weltzustände kausal oder anders verknüpfen. Prinzipiell könnte man auch die Verknüpfungen variabel halten und den Möglichkeitsspielraum durch Zustandsmerkmale der Welt begrenzen, die als generell und unveränderlich gelten. Es ist jedoch ebenso klar, daß dies nicht unserem normalen Verfahren entspricht. Als definitiv fixiert gelten die partikulären vergangenen Weltzustände, während die generellen Charakteristika dieser Zustände und relevanter Alternativen zu ihnen größtenteils variabel sind. (Definitiv fixiert im Bereich des physisch Möglichen sind auf der Zustandsebene vielleicht nur die Anzahl und Art der Elementarteilchen, aus denen die Welt sich gesetzlich konstituiert.) Vor allem aber steht ihre Fixierung nicht in Konkurrenz zu fixierten Gesetzen, sondern tritt zu diesen, wenn sie erfolgt, ergänzend hinzu. Und selbst wenn wir mit einem Begriff des ,,essentiell Möglichen“ (o.ä.) zu rechnen hätten, der völlig gesetzesunabhängig ist, würde sich für das praktische Können nichts ändern. Denn es ist allemal klar, daß dieses sich nicht darin erschöpfen kann, daß die betreffenden Handlungen die Rahmenbedingungen nicht verletzen, die das (wie immer spezifizierte) ,essentiell Mögliche" vorgibt. Ich lasse diese Deutungsmöglichkeit deshalb unberücksichtigt. 
rung, diese Alternativen stünden uns deshalb offen, weil sie nicht logisch unmöglich sind.

\section{Bedingungsspezifiziertes Können?}

Wenn es nun aber nicht das naturgesetzliche oder sonstige kausalgesetzliche Können ist, welches ist dann im Spiel? Nun, das Vorbild gewöhnlicher Dispositionsprädikate bietet noch einen zweiten Ansatzpunkt. Er betrifft jenes Können, das wir gewöhnlich als das der Fähigkeit oder Gelegenheit ansprechen. ${ }^{28}$ Äußeres Kennzeichen ist seine Graduierbarkeit. Die Zerbrechlichkeit von Glas ist z.B. nicht gleich. Während eine normale Scheibe einen Pistolenschuß nicht überlebt, widersteht ihm eine spezialbehandelte, dicke Panzerglasscheibe. Auch sie ist nicht absolut unzerbrechlich. Doch ihre Fähigkeit, zu zerbrechen, ist kleiner. Umgekehrt kann dasselbe Glasstück, je nach Gelegenheit, mehr oder weniger leicht zerbrechen. Ein Sektkelch in der Hand eines trunkenen Siegers geht leichter zu Bruch als einer in der Museumsvitrine.

28. Auf die Relevanz dieses Begriffs des Könnens verweist im übrigen nicht nur das Vorbild der Dispositionsprädikate. Austin (1956, a.a.O., 209ff.) hat gegen die Konditionalanalyse eingewandt, daß sie die Kontraposition des Satzes ,, kann $\mathrm{h}$ tun, wenn $\mathrm{x}$ h tun will“", nämlich ,,wenn $\mathrm{x}$ h nicht tun kann, will $\mathrm{x}$ h nicht tun", zulassen müsse, während diese in Wahrheit unzulässig sei. Dieser Einwand ist mißverständlich und öfter mißverstanden worden, hat jedoch seine Berechtigung. Austins Kritik ist zwar gegenstandslos, wenn man den kategorischen Satz ,, $\mathrm{x}$ kann $\mathrm{h}$ tun “ konsequent in den Konditionalsatz ,, $\mathrm{x}$ wird $\mathrm{h}$ tun, wenn $\mathrm{x}$ h will“" überführt (vgl. Davidson 1973, a.a.O., 67f.). Sie zeigt jedoch, da Austins Beobachtung fraglos korrekt ist, daß dies nicht der Sinn von ,,können“ sein kann, den wir dem Satz gewöhnlich zugrundelegen. Daß es sich nicht um einen verkappten Konditionalsatz handelt, wie Austin (a.a.O., 217) schlußfolgern wollte, wird dadurch allerdings nicht bewiesen. Der Grund für die fehlende Kontraponierbarkeit liegt vielmehr darin, daß das Können der Fähigkeit oder Gelegenheit unterstellt wird und daß dessen Fehlen das gleichzeitige Bestehen eines darüber hinausgehenden Wollens nicht ausschließt. Fähigkeit und Gelegenheit aber sind keine Eigenschaften, die losgelöst von Konditionalen zu explizieren wären, auch wenn sie (wie Austin richtig bemerkt hat, a.a. O., 229f.) nicht nur aus Konditionalen bestehen, sondern kategorische Präsuppositionen haben, sc. Aussagen über partiell erfüllte Kausalbedingungen (vgl. S. 223). 
Fähigkeit und Gelegenheit sind in der Regel gemeinsam dafür verantwortlich, daß wir die Disponiertheit von etwas höher oder geringer veranschlagen. Dabei können die Grenzen zwischen ihnen verwischen. Ein Beispiel zeigt das. Das Signal ,,Ready“ auf meinem Laserdrucker bedeutet, daß er jetzt drucken kann. Doch welcher Sinn von ,können“ ist hier im Spiel? In einem gewissen Sinn muß eine Maschine, die man als „Drucker“ bezeichnet, immer zum Drucken fähig sein, also auch wenn sie ausgeschaltet oder nicht angeschlossen ist. Legen wir diesen Fähigkeitssinn zugrunde, müssen wir „Ready" als Zeichen dafür verstehen, daß die Maschine jetzt die Gelegenheit hat, ihre generelle Druckfähigkeit auszuüben. Das eingelegte Papier, die Stromzufuhr, der Computeranschluß und die Aktivierung des Druckprogramms fungieren dann nicht als Bedingungen vorhandener oder gesteigerter Fähigkeit, sondern als Anwendungsbedingungen, die ein Können im Sinn der Gelegenheit spezifizieren. Ebensogut aber könnten wir sie als Bedingungen der situativen Druckfähigkeit der Maschine auffassen, und die Gelegenheit, diese Fähigkeit auszuüben, nur auf die Tatsache beziehen, daß eine Textdatei im Computer geladen ist und alle Druckbefehle bis auf das letzte ,Enter" erteilt sind. Oder wir könnten sagen, daß die Gelegenheit zum Druck nur durch das eingelegte Papier gebildet wird, während die übrigen Bedingungen durchweg der (situativen) Fähigkeit zugehören. Und so weiter.

Die Variabilität der Abgrenzung zeigt, daß der Rede von Fähigkeiten und Gelegenheiten derselbe Sinn von Können zugrundeliegt. Wie aber ist er genau zu verstehen? Offenbar haben wir es mit einer Erweiterung des natur- bzw. kausalgesetzlichen Könnens zu tun. Dieses bildet auch hier die Basis. Daß die Fähigkeit oder Gelegenheit zu etwas besteht, heißt zunächst nur, daß sein Eintreten mit den Naturgesetzen oder sonstigen kausalen Gesetzmäßigkeiten vereinbar ist. Das aber ist nicht alles. Hinzu kommt, implizit oder explizit, die Prämisse erfüllter Kausalbedingungen. Das Signal ,,Ready“ auf meinem Drucker oder der Aufkleber ,,Zerbrechlich“ auf einer Umzugskiste beziehen sich nicht nur darauf, daß das Zerbrechen des Kisteninhalts bzw. die Aktivität des Druckers physikalische Möglichkeiten sind, die unter bestimmten Bedingungen wirklich werden. Sie sagen auch, daß einige dieser Bedingungen schon erfüllt sind. Zur Abhebung gegenüber einer Rede vom Können, die aus- 
schließlich auf die Gesetzmäßigkeit abstellt, möchte ich hier deshalb von einem ,,bedingungsspezifizierten Können" sprechen. Umfang und Art der Spezifizierung können verschieden sein. Die Rede von „Fähigkeiten“ z.B. dürfte sich in der Regel auf die Erfülltheit innerer, die von ,,Gelegenheiten “ auf die Erfülltheit äußerer Bedingungen beziehen. Die Graduierung wiederum ist eine Folge davon, $\mathrm{da} B$ mehr oder weniger viele Bedingungen erfüllt sein können. Für das bedingungsspezifizierte Können allerdings gibt es hier eine obere Grenze. Sie ist erreicht, wenn alle Bedingungen bis auf eine erfüllt sind. Wird auch diese erfüllt, wird die Disposition aktualisiert; wir haben es nicht mehr mit einem Können zu tun, sondern mit einem kausalen Müssen.

Gewöhnliche Dispositionen sind immer bedingungsspezifiziert, äußerst selten jedoch, wenn jemals, in der Maximalform. Damit endet ihre Vorbildfunktion für das konditionale praktische Können. Denn dieses legt offensichtlich den Grenzfall maximaler Bedingungsspezifiziertheit zugrunde. So wie das Heilbleiben oder Zerbrechen einer Vase, gegebenfalls, nur noch an jenem seidenen Faden hängt, an dem ein Lampenschirm über ihr baumelt, so hängen Tun oder Unterlassen, gewissermaßen, am seidenen Faden des Wollens. Denn daß ein Mensch handeln kann, besagt nach der Konditionalanalyse, daß alle Bedingungen dafür, da $\beta$ er wirklich handelt, erfüllt sind außer der einen, daß er handeln will. Nur weil die ersteren, verpackt in den ,Restbedingungen“" unseres Schemas (K) (S. 211), pauschal vorausgesetzt werden. kann die Analyse sich auf die Affirmation der beiden Konditionalsätze beschränken.

Die diversen Anwendungsprobleme dieses Konzepts, die in der Analytischen Handlungstheorie extensiv diskutiert worden sind, übergehe ich hier. Mich interessiert die Rede vom ,Können“. Welchen Sinn hat sie im gegenwärtigen Fall? Bisher wissen wir nur, daß zwei Komponenten im Spiel sind, von denen die eine, der natur- oder kausalgesetzliche Sinn, für das praktische Können nichts hergibt (Abschnitt 7). Entscheidend ist also die zweite. Was besagt die Tatsache, daß alle Kausalbedingungen bis auf das Wollen erfüllt sind, für den Sinn des betreffenden Könnens? Formal bedeutet sie, daß der Spielraum des kausalgesetzlich Möglichen, der unzählige Alternativen umfaßt, situativ eingeengt ist bis auf zwei, sc. die willensabhängige Ausführung von h oder von 
-h. ${ }^{29}$ Die Gründe für diese Verengung bestimmen den Sinn, in dem wir die relevanten Teile der Welt für verschlossen und damit notwendig halten. Diese Gründe sind unschwer zu finden. Offenbar ist es die Faktizität der (definitiv) erfüllten Kausalbedingungen, die den Möglichkeitsspielraum verengt. Was unabänderlich feststeht, kann sich (in diesem Sinne von ,können“) nicht mehr anders verhalten. Darum allein kann der Konditionalanalytiker die ,,Restbedingungen" ignorieren. Was aber sind die Gründe, die ihn zugleich davon sprechen lassen, daß eine Bedingung, sc. die des Wollens und Nichtwollens von h, immer noch offen ist? Von diesen Gründen muß der spezifische Sinn des konditionalanalytischen , Könnens“ a abhängen. Was also sind sie?

Bei gewöhnlichen Dispositionen gibt es auch hier eine einfache Antwort. Der Grund dafür, daß eine Scheibe zerbrechen oder Zucker sich lösen kann, obwohl sie es faktisch nicht tun, liegt, abgesehen von der Vereinbarkeit dieser Ereignisse mit den Kausalgesetzen, vor allem darin, daß wir in der Lage sind, sie über relevante kausale Zwischenglieder handelnd herbeizuführen. Damit wird die Frage, welcher Sinn für das dispositionelle Können charakteristisch ist, auf das Handelnkönnen verschoben. $\mathrm{Ob}$ das auf alle Dispositionen zutrifft, kann man (trotz von Wright ${ }^{30}$ ) mit Grund bezweifeln,

29. Auf den ersten Blick ist die Situation paradox. Auf der einen Seite scheint eine Vergrößerung der Zahl erfüllter, relevanter Kausalbedingungen den als möglich ins Auge gefaßten Sachverhalt der Verwirklichung näher zu bringen, also insofern das Können zu steigern (vgl. S. 222f.). Auf der anderen Seite aber bedeutet sie, daß der Spielraum offener Alternativen kleiner wird und sich beim praktischen Können auf $z w e i$ verengt, sc. das Wollen und Nichtwollen der kritischen Handlung. Lassen wir uns dadurch jedoch nicht verwirren. Die Realisierungswahrscheinlichkeit muß vom Können unterschieden werden, und ob eine Vielzahl erfüllter Kausalbedingungen die Wahrscheinlichkeit wirklich erhöht, ist nicht ausgemacht. Es hängt allein davon $\mathrm{ab}$, ob die kritischen Bedingungen offen sind, und wenn ja, welche Wahrscheinlichkeit sie besitzen. Auch wenn es (z.B.) in einer bestimmten Situation nur noch auf meinen Willen ankommt, ob ich einen Mord begehe oder nicht, so wird dies, wenn ich es definitiv nicht will, ebenso sicher nicht geschehen, wie in einer Situation, in der ich weder die Fähigkeit noch die Gelegenheit habe, Menschen zu töten. Die Verengung des Spielraums erhöht das Gewicht der Bedingungen, die weiterhin offen sind; der Sinn der Rede vom Können oder Nichtkönnen aber wird davon nicht berührt.

30. G.H. v. Wright: Explanation and understanding, London 1971. 
braucht uns hier aber nicht zu kümmern. Denn es ist klar, daß diese Verschiebung beim praktischen Können ausscheidet. Hier geht es ja gerade darum, verständlich zu machen, worin das Handelnkönnen besteht. An dieser Stelle aber klafft eine konzeptionelle Lücke. Das Vorbild gewöhnlicher Dispositionsprädikate versagt, und die Konditionalanalyse selbst sagt nichts darüber, in welchem Sinne die kausale Bedingung des Wollens oder Nichtwollens offen ist.

\section{Die tieferen Gründe für das Scheitern der Analyse}

Damit ergibt sich folgendes Bild. Die konditionale Analyse des praktischen Könnens hat sich als unzureichend erwiesen, weil sie dem Urheberschaftskriterium nicht Rechnung trägt und das Problem der Willensfreiheit und das generelle Problem des Zustandekommens relevanter, handlungsbestimmender Zustände nicht löst (Abschnitt 5). Aber auch für das Können der Handlungsfreiheit ist sie nicht angemessen. Zwar kann sie das Kriterium der modalen Signifikanz erfüllen, vorausgesetzt daß man sie nach dem Muster gewöhnlicher, kontrafaktischer oder rein hypothetischer Dispositionsprädikate versteht (Abschnitt 6). Sie scheitert jedoch am Kriterium der modalen Spezifizierung (Abschnitte 7-8). Der kritische Punkt ist dabei nicht, daß sie den falschen Sinn von ,Können“ zugrundelegt oder überhaupt den Versuch macht, modalisierte auf nichtmodalisierte Sätze zurückzuführen. Die konditionale Analyse scheitert daran, daß sie den explikativ entscheidenden $S$ chritt nicht tut, sc. die Rede vom ,,Können“ zu spezifizieren. Insofern unterscheiden ihre Verfechter sich prinzipiell nicht von jenem Spaßvogel, der menschliche Freiheit auf das Prinzip ,Ab esse ad posse“ zu gründen sucht. So herausgefordert mag der Konditionalist die konzeptionelle Lücke offen eingestehen oder sich auf die heroische These versteifen, daß das praktische Können sich auf das kausalgesetzliche reduziert. Beides ist gleichermaßen ein Offenbarungseid. Von einem konditionalen Sinn des praktischen Könnens kann keine Rede sein. Wenn die Konditionalanalyse ihre modale Signifikanz behalten und nicht von vornherein auf ihren explikativen Anspruch verzichten will, muß sie auf einen Sinn von ,,Können“ zurückgreifen, den sie anderswo hernimmt. 
Woher aber kann die Ergänzung kommen? Die Iterierung der Konditionalanalyse auf beliebigen höheren Stufen des Wollens scheidet ebenso aus wie die Augustinische These, daß jedes Wollen in unserer Macht steht (S. 215f.). Niemand sollte auch auf den Gedanken verfallen, die epistemische Unbestimmtheit gewisser Teile der Welt für den Kern unserer Rede vom ,,praktischen Können“ zu halten. ${ }^{31}$ Denn dieser Vorschlag scheitert schon daran, daß sein Kriterium von kontrafaktischen Aussagen nicht erfüllt wird, bei denen der faktische Weltverlauf ex hypothesi als bekannt gilt. Deren Rede vom ,Können“ also würde das epistemisch interpretierte Konzept in keinem Fall explizieren können. Und es ist natürlich auch sonst, als ein nur epistemisch gültiges, höchst implausibel.

An dieser Stelle gibt es nur einen Ausweg. Wenn wir sagen, daß das Wollen und nachfolgende Handeln ebenso eintreten kann oder konnte wie das Wollen und nachfolgende Nichthandeln, meinen wir, antezedent oder im Rückblick auf einen relevanten früheren Zeitpunkt, daß beide Alternativen ontologisch offen sind, d.h. daß es nicht festliegt, welche von beiden eintritt. $\mathrm{Daß}$ dies die richtige Deutung sein muß, ergibt sich auch von der Gegenseite her. Denn wenn wir uns beim dispositionellen, bedingungsspezifizierten Können nicht nur auf offene Alternativen beziehen, sondern bestimmte Kausalbedingungen für erfüllt, also bestimmte Alternativen für verschlossen erklären, legen wir (wie oben angemerkt, S. 225) ebenfalls den Gesichtspunkt der Fixierung des Weltlaufs durch die schon bestehenden Fakten zugrunde. In exakt demselben Sinn, in dem die konditionale Analyse des praktischen Könnens bestimmte Alternativen durch die Fixierung der kausalen Restbedingungen ausschlie $\beta t, \mathrm{mu}$ sie die beiden verbliebenen als offene unterstellen, wenn sie denn an ihrem explikativen Anspruch festhalten will. Der Hintersinn des Shakespeare-Mottos, das ich dem Aufsatz vorangestellt habe, liegt darin, daß es auch dieses Ergebnis mitumfaßt. Denn der Protagonist des kurzen Dialogs will seinem Gegenüber vielleicht gar nicht die (prima facie implausible) Erklärung andienen,

31. Moore (1912, a.a.O., 94) hat dies seiner konditionalen Analyse als Zusatzkriterium angefügt. Allerdings war er sich der Tragweite dieses Kriteriums, wie seiner Analyse im ganzen, weit weniger sicher als spätere Vertreter eines epistemisch-indeterministischen Freiheitsbegriffs, wie etwa Popper und MacKay. 
daß man nicht tun kann, was man nicht will, sondern erklären, daß etwas, von dem bereits (wegen eines fixierten Wollens oder aus anderen Gründen) feststeht, daß man es nicht tun wird, nicht mehr zu den Alternativen gehört, die uns als Handelnden offenstehen.

In dieser Wendung liegt eine gewisse Ironie. Die Konditionalanalyse wurde eingeführt, um das freiheitsrelevante praktische Können mit der durchgängigen Determiniertheit der Jelt in Einklang zu bringen. Deshalb versuchte sie, es vom Kriterium der ontologischen Offenheit abzukoppeln. Das konnte solange als ein sinnvolles Vorhaben erscheinen, wie der modale Sinn der verwendeten Konditionale unspezifiziert blieb. Stellt man sich dieser Aufgabe jedoch und expliziert den Sinn so, wie es dem Vorbild des dispositionellen, bedingungsspezifizierten Könnens entspricht, wird klar, daß die konditionale Analyse selbst das voraussetzt, was sie verabschieden wollte. Wir können sie deshalb, nachdem wir den explikativen Zirkel - geistesgeschichtlich wie systematisch - durchlaufen haben, hinter uns lassen und uns der Sache selbst zuwenden. 